\title{
Abstract: Automatic Plane Adjustment in Surgical Cone Beam CT-volumes
}

\author{
Celia Martín Vicario $^{1}$, Florian Kordon ${ }^{1,2,3}$, Felix Denzinger ${ }^{1,2}$, \\ Markus Weiten ${ }^{2}$, Sarina Thomas ${ }^{5}$, Lisa Kausch ${ }^{5}$, Jochen Franke ${ }^{6}$, Holger Keil ${ }^{7}$, \\ Andreas Maier ${ }^{1,3,4}$, Holger Kunze ${ }^{2,1}$ \\ ${ }^{1}$ Pattern Recognition Lab, Universität Erlangen-Nürnberg (FAU), Erlangen \\ ${ }^{2}$ Siemens Healthcare GmbH, Forchheim \\ ${ }^{3}$ Erlangen Graduate School in Advanced Optical Technologies (SAOT), Universität \\ Erlangen-Nürnberg (FAU), Erlangen \\ ${ }^{4}$ Machine Intelligence, Universität Erlangen-Nürnberg (FAU), Erlangen \\ ${ }^{5}$ Division of Medical Image Computing, German Cancer Research Center, Heidelberg \\ ${ }^{6}$ Department for Trauma and Orthopaedic Surgery, BG Trauma Center \\ Ludwigshafen, Ludwigshafen \\ ${ }^{7}$ Department of Trauma and Orthopedic Surgery, University Hospital Erlangen, \\ Universität Erlangen-Nürnberg (FAU), Erlangen \\ Celia.Martin@fau.de
}

Cone beam computed tomography (CBCT) is used intra-operatively to assess the result of surgery. Due to limitations of patient positioning and the operating theater in general, the acquisition usually cannot be performed such that the axis-aligned multiplanar reconstructions (MPR) of the volume match the anatomically oriented MPRs. This needs to be corrected manually, which is a time-consuming and complex task and requires the surgeon to interact with non-sterile equipment. To this end, this study investigates a fully-automatic solution to directly regress the standard plane parameters from a CBCT volume of the calcaneus and ankle regions. A PoseNet convolutional neural network $(\mathrm{CNN})$ is adapted and trained, comparing a 6D-, Euler angle- and quaternionbased approach to represent the plane rotation [1]. In addition, a cost function is optimized to incorporate orientation constraints. The best-performing CNN - which uses the $6 \mathrm{D}$ representation - estimates the plane normal with a median accuracy of $5^{\circ}$, the in-plane rotation with a median accuracy of $6^{\circ}$, and the position with a median accuracy of $6 \mathrm{~mm}$. The inference time is less than $0.05 \mathrm{~s}$.

\section{References}

1. Martin Vicario C, Kordon F, Denzinger F, et al. Automatic plane adjustment of orthopedic intraoperative flat panel detector CT-volumes. Proc MICCAI. 2020; p. 486-495. 